\title{
DIAGNÓSTICO Y MANEJO PRECOZ DE LA GANGRENA DE FOURNIER*
}

\author{
Drs. Felipe Castillo H. ${ }^{1}$, Javier Moraga C. ${ }^{2,3}$, Pablo Pérez C. ${ }^{1}$, \\ Carlos Álvarez Z. ${ }^{2}$, Andrés Iglesias B. ${ }^{2}$ \\ 1 Servicio de Cirugía Hospital Barros Luco-Trudeau, Universidad de Chile. \\ 2 Servicio de Urgencias Hospital Barros Luco-Trudeau. \\ 3 Universidad Autónoma de Chile. \\ Santiago, Chile.
}

\section{Abstract \\ Early diagnosis and management of Fournier's gangrene}

Aim: The aim of this study is to report the results of a series of patients diagnosed with Fournier's gangrene underwent surgical debridement plus broad-spectrum antibiotics in the emergency department of the Hospital Barros Luco-Trudeau (HBLT) between 2009 and 2013, in terms of mortality associated with the disease. Material and Methods: Between 2009 and 2013, a case series of patients with diagnosis of Fournier's gangrene treated in the Emergency Department of the Hospital Barros Luco-Trudeau. The outcome variable was mortality attributed to the disease (MAD). Other variables were: age, sex, comorbidities, focus of origin, waiting time for antibiotic treatment and surgery to start, number of surgical debridement and agents isolated from cultures. Descriptive statistics were used, with calculation of measures of central tendency and dispersion. Results: During the study period, 56 patients were identified with diagnosis of Fournier's gangrene ( $60.7 \%$ were male) with a mean age of 52 years (23-75 years old). The MAD was $48.2 \%$. The most common comorbidity was diabetes $(66.6 \%)$. The more prevalent focus of origin was anorectal pathology $(42.9 \%)$. The average waiting time from diagnosis to initiation of antibiotic therapy and surgery was 40 minutes (15-80) and 580 minutes (20-4320), respectively. The required surgical debridement average was 4 . Isolated on the intraoperative tissue cultures agent was E. coli (51.8\%). Conclusion: Mortality attributable to Fournier's gangrene is similar to that observed in the literature.

Key words: "Fournier gangrene" [MeSH], "mortality" [MeSH], "fasciitis, necrotizing" [MeSH].

\section{Resumen}

Objetivo: El objetivo de este estudio es comunicar los resultados observados en una serie de pacientes con diagnóstico de gangrena de Fournier sometidos a aseo quirúrgico con debridamiento más terapia antibiótica de amplio espectro, en el Servicio de Urgencias del Hospital Barros Luco-Trudeau (HBLT), entre los años 2009 y 2013, en términos de mortalidad asociada a la enfermedad. Material y Método: Serie de casos

*Recibido el 11 de junio de 2014 y aceptado para publicación el 28 de julio de 2014.

Los autores no refieren conflictos de interés.

Correspondencia: Dr. Javier Moraga C.

Javier.moragac@gmail.com 
de pacientes con diagnóstico de gangrena de Fournier tratados en el Servicio de Urgencias del Hospital Barros Luco-Trudeau entre 2009 y 2013. La variable resultado fue mortalidad atribuida a la enfermedad (MAE). Otras variables de interés fueron: edad, sexo, patologías asociadas, foco de origen, tiempo de espera para el inicio del tratamiento antibiótico y cirugía, número de aseos y agentes aislados en los cultivos. Se utilizó estadística descriptiva, con cálculo de medidas de tendencia central y dispersión. Resultados: En el período en estudio se identificaron 56 pacientes con diagnóstico de Gangrena de Fournier (60,7\% eran masculinos), con un promedio de edad de 52 años (23-75 años). La MAE fue 48,2\%. La patología asociada más frecuente fue la diabetes $(66,6 \%)$. El foco de origen más prevalente fue la patología anorrectal $(42,9 \%)$. El tiempo promedio de espera desde el diagnóstico hasta el inicio de la terapia antibiótica y la cirugía fue de 40 minutos (15-80) y 580 minutos (20-4320) respectivamente. El promedio de aseos requeridos fue de 4 . El agente más aislado en los cultivos de tejido intraoperatorio fue Escherichia coli (51,8\%). Conclusión: La mortalidad atribuible a la Gangrena de Fournier es similar a la observada en la literatura.

Palabras clave: Gangrena de Fournier, mortalidad, fascitis necrotizante.

\section{Introducción}

La gangrena de Fournier fue inicialmente descrita por Jean Alfred Fournier en 1883, como una gangrena genital idiopática fulminante en varones sanos. Actualmente, la gangrena de Fournier se describe como una fascitis necrotizante de la región genitoperineal, que ocurre tanto en hombres como en mujeres y que es provocada por una infección polimicrobiana sinérgica entre microorganismos aerobios y anaerobios ${ }^{1}$.

El foco de origen más frecuentemente precisado es el colorrectal, aunque puede atribuirse también a focos cutáneos, ginecológicos o urológicos ${ }^{2}$, de mayor prevalencia alrededor de la quinta década de la vida y que afecta más a hombres que a mujeres, en una relación 6:1 según algunas series ${ }^{3}$.

Existe gran cantidad de factores predisponentes, dentro de los que destacan la diabetes mellitus, el alcoholismo y la inmunodepresión de diferentes causas ${ }^{4}$.

Se han descrito nuevos tratamientos para esta patología, como por ejemplo, el oxígeno hiperbárico ${ }^{5}$. Sin embargo, el tratamiento que ha demostrado los mejores resultados medidos en términos de mortalidad asociada a la enfermedad es el aseo quirúrgico precoz con desbridamiento agresivo asociado a antibióticos de amplio espectro ${ }^{6}$. Un tema que genera controversia es el uso de colostomía derivativa, procedimiento recomendado por algunos grupos y rechazado enfáticamente por otros ${ }^{7}$.

La mortalidad de esta patología ha sido reportada entre 0 y $80 \%$, dependiendo las series ${ }^{8,9}$.

El presente manuscrito fue escrito siguiendo la pauta de chequeo STROBE para estudios observacionales ${ }^{10}$.

El objetivo de este estudio es comunicar los resultados observados en una serie de pacientes con diagnóstico de gangrena de Fournier sometidos a aseo quirúrgico con desbridamiento más antibióticos de amplio espectro en el Servicio de Urgencias del Hospital Barros Luco-Trudeau (HBLT), entre los años 2009 y 2013, en términos de mortalidad asociada a la enfermedad.

\section{Material y Método}

\section{Diseño}

Serie de casos retrospectiva.

\section{Centro}

El estudio fue realizado en el Servicio de Urgencias del HBLT, entre el año 2009 y 2013, ambos incluidos.

\section{Población}

Muestra no probabilística de pacientes con diagnóstico de gangrena de Fournier sometidos a aseo quirúrgico y antibióticos en el Servicio de Urgencias del HBLT. No se excluyeron pacientes.

\section{Tratamiento}

El tratamiento consistió en aseo quirúrgico con desbridamiento asociado al uso de antibióticos de amplio espectro (Ceftriaxona y Metronidazol).

\section{Maniobra}

Se realizó una revisión de carácter retrospectiva de los registros de atención del Servicio de Urgencia del HBLT entre los años 2009 y 2013, período en que se identificó a los pacientes con diagnóstico de gangrena de Fournier sometidos al tratamiento antes señalado.

\section{Variables en estudio}

La variable resultado fue mortalidad atribuible a la gangrena de Fournier durante el período en estudio. Otras variables de interés fueron: edad, sexo, patologías asociadas, foco de origen, tiempo 
de espera para el inicio del tratamiento antibiótico y cirugía, necesidad de aseos extras y agentes aislados en los cultivos.

\section{Sesgos}

Los datos fueron recogidos de forma enmascarada con el objetivo de reducir posibles sesgos de medición y reporte.

\section{Tamaño de la muestra}

Por tratarse de un estudio de carácter descriptivo no se realizó cálculo del tamaño de la muestra.

\section{Métodos estadísticos}

Se realizó un análisis exploratorio de los datos. Posteriormente, se utilizó estadística descriptiva de las variables con cálculo de medidas de tendencia central y dispersión. La tabulación y presentación de los datos se realizó con el software MS Excel 2007.

\section{Aspectos éticos}

Los datos obtenidos de la revisión fueron cuidadosamente codificados con el fin de resguardar la confidencialidad de los pacientes. Los autores declaran no tener conflictos de interés con los resultados obtenidos.

\section{Financiamiento}

El presente estudio no contó con fuentes formales de financiamiento.

\section{Resultados}

Durante el período en estudio se identificaron 56 pacientes diagnosticados con gangrena de Fournier en el Servicio de Urgencia del HBLT; de ellos, 34 $(60,7 \%)$ eran masculinos, con un promedio de edad de 52 años (23-75 años).

Las patologías asociadas se observan en la Tabla 1. El foco de origen más prevalente fue la patología anorrectal, con 42,9\% (Tabla 2).

El tiempo promedio de espera desde el diagnóstico hasta el inicio de la terapia antibiótica y la cirugía fue de 40 minutos (15-80) y 580 minutos (20-4.320) respectivamente. El $42,9 \%$ de los pacientes requirió más de un aseo quirúrgico; el promedio de estos últimos fue de 4 aseos.

En el 51,8\% de los cultivos de tejido intraoperatorio, el agente aislado fue Escherichia coli, mientras que el restante $48,2 \%$ de los cultivos fue polimicrobiano.

La mortalidad global durante el período en estudio fue de $62,5 \%$, y la atribuible a la gangrena de Fournier fue de un 48,2\%.

\section{Discusión}

La gangrena de Fournier es una enfermedad compleja que requiere de manejo en una unidad de cuidados intensivos o, a lo menos, intermedios, por un equipo multidisciplinario compuesto por cirujanos, intensivistas, urólogos, ginecólogos, nutriólogos, cirujanos plásticos e infectólogos, además de un equipo de enfermería con experiencia en el aseo y curación de este tipo de lesiones. Lo anterior representa un gran desafío tanto para cirujanos como para otros profesionales involucrados en su tratamiento, no sólo por la agresividad de los patógenos involucrados, sino también porque presenta una baja incidencia, lo que hace que el entrenamiento, tanto en los cuidados quirúrgicos como los cuidados post operatorios, sea complejo para quienes se forman en esta área ${ }^{2,11}$.

El promedio de edad de los pacientes de la serie revisada fue de 52 años, con valores extremos de 23 y 75 años; asimismo, alrededor del $60 \%$ de los pacientes fueron de sexo masculino, lo que se condice con lo observado en diversas series ${ }^{12-14}$.

En relación con los factores predisponentes o patologías asociadas de los pacientes, podemos observar que la diabetes es la más frecuente, seguida de otras, como tabaquismo e hipertensión arterial, al igual como se evidencia en la literatura ${ }^{7,12-14}$.

Si bien es difícil precisar el foco de origen, en nuestra serie se evidenció que la patología anorrectal fue predominante, con un $43 \%$, frente a otras causas, como cutáneas, urológicas o idiopáticas, lo que concuerda con lo expuesto en otras series ${ }^{15-16}$.

Tabla 1. Patologías asociadas

\begin{tabular}{|lcc|}
\hline Patologías asociadas & n & $\mathbf{\%}$ \\
\hline Diabetes & 37 & 66,6 \\
\hline Tabaquismo crónico & 27 & 47,6 \\
\hline Hipertensión arterial & 24 & 42,8 \\
\hline Obesidad & 13 & 23,8 \\
\hline Enfermedad renal crónica Etapa V & 11 & 19,6 \\
Postración & 7 & 12,5 \\
\hline
\end{tabular}

Tabla 2. Focos de origen

\begin{tabular}{|lcc|}
\hline Foco de origen & n & \% \\
Anorrectal & 24 & 42,9 \\
Cutáneo & 16 & 28,6 \\
Urinario & 11 & 19,6 \\
Idiopático & 5 & 8,9 \\
\hline
\end{tabular}


El tiempo promedio entre el diagnóstico y la cirugía fue de aproximadamente $9 \mathrm{~h}$. Respecto de este punto, existe controversia. Mientras para algunos autores esta es la variable determinante en el pronóstico de la enfermedad ${ }^{1}$, para otros no constituiría un factor de riesgo significativo ${ }^{12}$. El 42,9\% de los pacientes requirió más de un aseo quirúrgico, el número de aseos realizados a los pacientes fue de 4 , en promedio. Esto podría estar determinado por aseos iniciales con escasos márgenes de seguridad, que según algunos autores debería ser de a lo menos $6 \mathrm{~cm}$ más allá de los bordes con sangrado activo y tejido celular subcutáneo aparentemente sano ${ }^{12}$. Otro punto importante de destacar es que todos los pacientes recibieron cobertura antibiótica con Ceftriaxona y Metronidazol, con el fin de cubrir bacterias Gram positivos, negativos y anaerobios. No se requirió de colostomías en esta serie, lo que se condice con lo expresado por parte de la literatura. Sin embargo, esto continúa siendo motivo de discusión entre diferentes autores ${ }^{2}$.

En relación con los patógenos aislados, el más frecuente fue Echerichia coli, con 51,8\%, y el 48,2\% de los cultivos fue polimicrobiano. Estos valores son similares a los descritos en la literatura ${ }^{13}$.

La mortalidad atribuida a la gangrena de Fournier en la serie revisada fue de $48,2 \%$, valor que se encuentra dentro de los limites de mortalidad expresados en la literatura ${ }^{8-9}$. Sin embargo, fue mayor que lo evidenciado en otras series nacionales ${ }^{2,16}$.

El presente estudio intenta describir los resultados del tratamiento de la gangrena de Fournier, en términos de mortalidad asociada a la enfermedad, obtenidos a partir de una serie de pacientes tratados en el Servicio de Urgencias del HBLT. Somos conscientes de las limitaciones del estudio, algunas de las cuales dicen relación con sesgos de medición y de reporte que son inherentes al diseño del mismo. $\mathrm{Si}$ bien los resultados obtenidos presentan validez interna para con los pacientes del estudio, estos resultados carecen de validez externa o, en otras palabras, no pueden ser extrapolados a la población; por otra parte, este tipo de diseño no nos permite realizar asociaciones estadísticas entre las variables. Es por lo anteriormente expuesto que se requiere de estudios de mejor nivel de evidencia, como ensayos clínicos aleatorizados que nos permitan evaluar no sólo asociaciones entre las variables, sino también objetivar la magnitud del efecto del tratamiento.

\section{Referencias}

1. Norton K, Johnson L, Perry T, Perry K, Sehon J, Zibari G. Managment of Fournier's Gangrene: An Eleven Year
Retrospective Analysis of Early Recognition, Diagnosis, and Treatment. Am Surg. 2002;68:709-13.

2. Bocic G, Abedrapo M, Azolas R, Llanos J. Enfermedad de Fournier. Tratamiento. Rev Chil Cir. 2010;62:635-8.

3. Ghnnam W. Fournier's gangrene in Mansoura Egypt a review of 74 casos. J Postgrad Med. 2008;54:1069.

4. García A, Acuña J, Gutiérrez J, Martínez R, Gómez L. Gangrena de Fournier: nuestra experiencia en 5 años, revisión de la literatura y valoración del índice de severidad de la Gangrena de Fournier. Arch Esp Urol. 2009;62:532-40.

5. Janane A, Hajji F, Ismail T, ChafiquiJ, Ghadouane M, Ameur A, et al. Terapia de oxígeno hiperbárico complementaria al desbridamiento en el control de la Gangrena de Fournier: Utilidad de la puntuación de un índice de severidad en la predicción de la gravedad de la enfermedad y la supervivencia del paciente. Actas Urol Esp. 2011;35:332-8.

6. Montoya R, Izquierdo E, Nicolae B, Pellicer E, Aguayo J, Miñana B. Gangrena de Fournier. Análisis descriptivo de 20 casos y revisión de la bibliografía científica. Actas Urol Esp. 2009;33:873-80.

7. Bannura G. Enfermedad de Fournier. Rev Med Chile 1992;120:304-10.

8. Pizzorno R, Bonini F, Donelli A, Stubinski R, Medica $\mathrm{M}$, Carmignani G. Hyperbaric oxygen therapy in the treatment of Fournier's disease in 11 male patients. J Urol. 1997;158:837-40.

9. Garcés C, Gómez C, Flores I, Muñoz J. Gangrena de Fournier en asociación con el uso de anti-inflamatorios no esteroideos. Reporte de un caso pediátrico. Rev Chil Infect. 2010;27:341-4.

10. STROBE Statement. Strengthening the reporting of observational studies in epidemiology. Disponible en http://www.strobe-statement.org [Consultado el 31 de mayo de 2014].

11. Paty R, Smith A. Gangrene and Fournier's Gangrene. Urol Clin North Am. 1992;19:149-62.

12. Azolas R. Factores de riesgo para mortalidad en gangrena de Fournier. Rev Chil Cir. 2011;63:270-5.

13. Bocic G, Jensen C, Cáceres M, Garrido R, Cúneo A, Abedrapo M, et al. Enfermedad de Fournier: Técnica de tratamiento modificada, once años de experiencia clínica. Rev Chil Cir. 2003;55:232-8.

14. Jensen C, Azolas C, Pérez G, Vergara J, Garrido R, Cárdenas R. La gangrena perineal. Nuestra experiencia en el manejo de veintiún casos. Rev Chil Cir. 1992;44:614.

15. Bannura G, Vera E, Schultz M, Aguayo P, Espinoza M. Gangrena de Fournier. Rev Chil Cir. 1992;44:289-93.

16. Ocares M, Bravo J, González R, Madariaga J. Gangrena Perineal: Enfermedad de Fournier. Rev Chil Cir. 2002;54:517-24. 